\title{
Identification of complex septic odorants in Huangpu River source water by combining the data from gas chromatography-olfactometry and comprehensive two-dimensional gas chromatography using retention indices
}

\author{
Qingyuan Guo ${ }^{a}$, Jianwei Yu ${ }^{\mathrm{a}, *}$, Kai Yang ${ }^{\mathrm{a}}$, Xiaodong Wen ${ }^{\mathrm{a}, \mathrm{b}}$, Haifeng Zhang ${ }^{\mathrm{a}}$, Zhiyong Yu ${ }^{\mathrm{a}}$, Hongyan $\mathrm{Li}^{\mathrm{a}}$, \\ Dong Zhang ${ }^{\mathrm{c}}$, Min Yang ${ }^{\mathrm{d}}$ \\ a Key Laboratory of Drinking Water Science and Technology, Research Center for Eco-Environmental Sciences, Chinese Academy of Sciences, Beijing 100085, China \\ b School of Chemical and Environmental Engineering, China University of Mining and Technology, Beijing 100085, China \\ c Shanghai National Engineering Research Center of Urban Water Resources Co., Ltd., Shanghai 200082, China \\ d State Key Laboratory of Environmental Aquatic Chemistry, Research Center for Eco-Environmental Sciences, Chinese Academy of Sciences, Beijing 100085, China
}

\section{H I G H L I G H T S}

- Septic odorants were identified by combining the data from GC-O/MS and GC $\times$ GC-TOFMS

- OAV was adopted to evaluate septic and musty odorants in drinking water

- Bis(2-chloroisopropyl) ether, DEDS and DMDS were major septic odorants in HP River

\section{G R A P H I C A L A B S T R A C T}

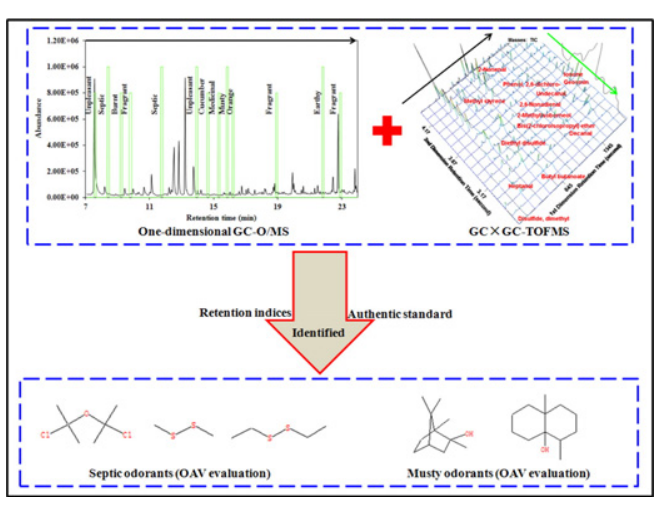

\section{A B S T R A C T}

Identification of the trace odorants causing the septic odors in source waters with complex matrixes has long been a big challenge. The Huangpu (HP) River, an important source water for Shanghai, has long been suffering from septic and musty odors, although major odorants have not been identified. In this study, combining the data from gas chromatography-olfactometry with mass spectrometry (GC-O/MS) and comprehensive twodimensional gas chromatography with time-of-flight mass spectrometry (GC $\times$ GC-TOFMS) using retention indices (RIs) was used for the identification of odorants in HP source water. Olfactometry peaks detected in water extracts by GC-O/MS were combined with the chromatography peaks detected by GC $\times$ GC-TOFMS based on the RIs determined using the retention times (RTs) of alkanes C7-C30. A total of thirteen olfactometry peaks were obtained though GC-0/MS analysis, and potential odorants corresponding to each of the olfactometry peaks were screened based on the odor characteristics and match similarity using GC $\times$ GC-TOFMS. Finally, fourteen odorants (one odorant was detected in GC $\times$ GC-TOFMS without an olfactometry peak), including three septic odorants (bis(2-chloroisopropyl) ether, diethyl disulfide and dimethyl disulfide) and two musty ones (geosmin and 2-MIB), were confirmed by using authentic standards. The septic and musty odorants in six

\footnotetext{
* Corresponding author.

E-mail address: jwyu@rcees.ac.cn (J. Yu).
} 
source water samples taken over a period of six months were quantified. Bis(2-chloroisopropyl) ether, with an odor activity value (OAV) of 1.84-3.2, was found to be a major septic odorant in HP source water, followed by diethyl disulfide (OAV 1.56-1.96) and dimethyl disulfide (OAV 0.37-2.42), while geosmin (OAV 4.37-11.44) was the major musty odorant, followed by 2-MIB (OAV 1.13-1.89). This is the first comprehensive study focusing on the identification of odorants in a complex source water. The integrated approach used in this study could be applied for the identification of odorants in other complex source waters suffering similar odor problems.

(c) 2016 Elsevier B.V. All rights reserved.

\section{Introduction}

Odor problems have been a major concern for drinking water in many countries (Vaughn, 1967; Suffet et al., 1999; Yang et al., 2008). Among different types of odors, septic and musty/earthy odors are the ones most frequently reported worldwide (Suffet et al., 1999; Watson, 2004; Yang et al., 2008). In comparison with musty/earthy odors usually triggered by natural origins from algal or fungi metabolites including 2methylisoborneol (2-MIB) and geosmin (Lin et al., 2002; Chen et al., 2010; Sun et al., 2013), septic odor is much more difficult to tolerate for consumers because of its perception as disgusting, irritating and its anthropogenic pollution origin (Vaughn, 1967; Suffet et al., 2004), requiring a quick response from water suppliers. However, the identification of septic odorants has long been a big challenge because of the diverse potential odorants derived from different origins including algal or fungi metabolism, domestic or industrial pollution, etc. (Tomar and Abdullah, 1994; Young et al., 1996; Watson, 2004). Thioethers (Yu et al., 2009), thiols (Schiffman et al., 2001), pyrazines (Young et al., 1996), pyridines (Pandey et al., 2007), phenols (Davi and Gnudi, 1999) and indoles (Hwang et al., 1995) have been reported to cause septic-like odors even at a level of ng/L to $\mu \mathrm{g} / \mathrm{L}$.

The Huangpu (HP) River, an important source water for Shanghai, has long been suffering from septic and musty odors because of water pollution (Ren et al., 2003; Yang et al., 2007; Wang et al., 2014). By utilizing gas chromatography-olfactometry with mass spectrometry (GC-O/MS), the musty/earthy odors in the HP River have been identified as associated with 2-MIB and geosmin (Yu et al., 2009; Sun et al., 2013). As for the septic odor, dimethyl trisulfide (DMTS) was detected in HP source water during a water quality survey (Yu et al., 2009). However, the use of one-dimensional gas chromatography (1D GC) has limited the GC-O/MS system in terms of separation capacity and resolution (Dalluge et al., 2003), which makes it difficult to identify the odorants in complex samples. To identify odorants effectively, the data from comprehensive two-dimensional gas chromatography with time-of-flight mass spectrometry (GC $\times$ GC-TOFMS) (Adahchour et al., 2003; d'Acampora Zellner et al., 2008; Chin et al., 2011), which has a high chromatographic resolution for the identification of complex odorants, has been combined with the data from GC-O/MS in the food industry. By using this approach, various odorants in wine, coffee and fruit samples have been successively identified (Eyres et al., 2005; Chin et al., 2011).

In this study, a strategy to combine the data from GC-O/MS and GC $\times$ GC-TOFMS using the retention indices (RIs) of alkanes was applied in the identification of the major odorants in HP River source water. GC-O/MS was used to obtain olfactometry peaks for the water sample, and GC $\times$ GC-TOFMS was used to measure compound peaks. Then the potential odorants were selected by combining the data from the two instruments using RIs, and confirmed on GC $\times$ GC-TOFMS using authentic standards. Four pre-concentration methods, including liquid-liquid extraction (LLE), solid phase extraction (SPE), solid phase micro-extraction (SPME) and simultaneous distillation extraction (SDE), were compared in terms of their ability to acquire the numbers of olfactometry peaks observed in GC-O/MS. To verify the correspondence between the olfactometry peaks in GC-O/MS and the corresponding chromatography peaks in GC $\times$ GC-TOFMS, four typical odorants including 2-methoxy-3-(2-methylethyl)-pyrazine (IPMP), 2-methoxy3-(2-methylpropyl)-pyrazine (IBMP), 2-methylisoborneol (2-MIB) and geosmin were added to a solution of alkanes. After GC-O/MS analysis, potential odorants in the corresponding olfactometry regions were screened based on their odor characteristics and similarity using GC $\times$ GC-TOFMS, and confirmed by using authentic standards. The septic and musty odorants in six source water samples taken over a period of six months were quantified, and their odor activity values (OAVs), which were acquired by dividing the odorant concentration by the odor threshold concentration (OTC), were calculated. This is the first study to combine the data from GC-O/MS and GC $\times$ GC-TOFMS for the identification of potential odorants in a highly complex water matrix.

\section{Materials and methods}

\subsection{Chemicals and reagents}

A total of 31 authentic standards including heptanal, cyclohexanone, 2-ethyl-pyridine, 3-ethyl-pyridine, diethyl disulfide, 2-chloro-pyridine, 2,4-dimethyl-pyridine, 2-cyclohexen-1-one, $\alpha$-methyl-styrene, butyl butanoate, 1-octen-3-one, 1-octen-3-ol, bis(2-chloroisopropyl) ether, 2-methyl-phenol, 2-nonenal, o-cymene, isophorone, 2,6-nonadienal, 2,1,3-benzothiadiazole, 2,6-dichloro-phenol, ethyl benzoate, 2decanone, 3-phenyl-2-propenal, 2-methyl-quinoxaline, 1,7-dimethylnaphthalene, 2-phenyl-pyridine, 2-methylisoborneol, decanal, undecanal, geosmin and ionone were selected for the confirmation of potential odorants, and purchased at the highest level of purity from Sigma-Aldrich (USA). Stock solutions of $1000 \mathrm{mg} / \mathrm{L}$ for authentic standards were prepared by diluting different standard solutions with HPLC grade methanol. A mixed standard of alkanes (C7-C30) for determination of RIs was purchased at analytical grade purity from Supelco Co. (USA). Solvents including acetone, methanol and methylene chloride of HPLC grade were purchased from Fisher Scientific Co. (China). Reagents including $\mathrm{NaCl}$ and anhydrous $\mathrm{Na}_{2} \mathrm{SO}_{4}$ were purchased at guaranteed reagent purity grade from Beijing Chemicals Ltd. (China), which were heated to $450{ }^{\circ} \mathrm{C}$ for $2 \mathrm{~h}$ before use. Ultrapure deionized water $(18 \mathrm{M} \Omega * \mathrm{~cm}$ ) was produced with a Milli-Q purification system.

\subsection{Sampling and sample preparation}

Six water samples were taken from the water intake of a waterworks of Shanghai located in the lower HP River during a period from July to December in 2014. Once each month, $1 \mathrm{~L}$ water was collected in an amber glass bottle with no head space, and then transported to the laboratory immediately in an insulated box with ice bags to maintain the temperature below $4{ }^{\circ} \mathrm{C}$. All sampling tap and bottles were fully rinsed prior to taking samples.

Sensory characteristics of the water samples were evaluated using flavor profile analysis (FPA) (APHA, 2005) immediately once received, and the pre-concentration for GC-O/MS and GC $\times$ GC-TOFMS analyses was completed within $24 \mathrm{~h}$. Prior to pre-concentration, the samples were first filtered through glass-fiber filters (GF/C, $1.2 \mu \mathrm{m}$, Whatman, UK). Methylene chloride (HPLC grade) was used for liquid-liquid extraction (LLE). The LLE pre-concentration process was performed as follows: $500 \mathrm{~mL}$ water samples were extracted using methylene chloride twice $(50 \mathrm{~mL}$ and $30 \mathrm{~mL}$ methylene chloride for the first and second extractions, respectively), then dehydration was carried out with $\mathrm{Na}_{2} \mathrm{SO}_{4}$ (guaranteed reagent). Samples were then concentrated to a 
final volume of $100 \mu \mathrm{L}$, following rotary evaporation and blowing off under a gentle nitrogen stream. The pressure of the rotary evaporation chamber was 920 mbar, the temperatures of the water bath and recirculated cooling water were $28^{\circ} \mathrm{C}$ and $10{ }^{\circ} \mathrm{C}$, respectively. To compare the efficiency of the pre-concentration methods, several other pre-concentration methods including simultaneous distillation extraction (SDE) using methylene chloride (Young and Suffet, 1999), solid phase micro-extraction (SPME) using fibers coated by CW/PDMS, PDMS/DVB, or CAR/PDMS/DVB (Watson et al., 1999), and solid phase extraction (SPE) using HLB and C18 cartridges (Wright et al., 2014) were also conducted. The experimental procedures of the other three extraction methods can be found in Supporting Information.

\subsection{Sensory analysis}

FPA was used to evaluate the odor characteristics of water samples; a detailed description of the training and application procedures can be found in the Standard Methods for Water and Wastewater (APHA, 2005). 3-4 non-smokers between 24 and 45 years of age with normal olfactory function and no known anosmia were selected as the panelists. Seven-point scales of 1-12 were used to describe the intensity of samples (1: odor threshold, 2 \& 4: soft odor intensity, 6 \& 8: moderate odor intensity, 10 \& 12: strong odor intensity). The panel conducting FPA also analyzed the sample extracts passed through GC-O/MS.

The 3-alternative forced choice (3-AFC) method was used for determining the odor threshold concentration (OTC) of odorants (ASTM, 1997). While conducting 3-AFC analysis, several series were prepared, which included two controls and one spiked sample in each series. The controls were adopted blanks consisting of ultrapure deionized water without odor, the spiked standard was diluted in ultrapure deionized water and the spiked ultrapure deionized water was added to the blank as a spiked sample. Six panelists were presented with eighteen conical flasks, corresponding to six spiked sample levels, with each level differing by a dilution factor of $2(\mathrm{X} / 64, \mathrm{X} / 32, \mathrm{X} / 16, \mathrm{X} / 8, \mathrm{X} / 4$ and $X / 2$ ) and were evaluated in ascending order beginning from the most diluted one. All conical flasks were labeled with one of 3 randomized letters $(A|B| C)$. Panelists were instructed to smell and choose the spiked sample in each set of three flasks, and they would have to guess one of them if they could not perceive a difference (forced choice). The final OTC was averaged among all panelists.

\subsection{GC-O/MS analysis}

During the GC-O/MS analysis, an olfactory detector port (Gerstel, Germany) was used to record the characteristic olfactometry peaks, including odor descriptors and intensities, and quadrupole MS (Agilent 5975, Agilent Technologies, USA) was adopted for chromatography peaks. Odor descriptors were verbalized and recorded while olfactometry peaks eluted during the mass spectrometry runs. The odor intensity of each olfactometry peak was marked using a controller device with a score from 1 to 4 ( $1=$ low; $2=$ medium; $3=$ high; $4=$ very high) (van Ruth, 2001). Each analyst had at least $4 \mathrm{~h}$ separation between runs to avoid fatigue and contamination.

Sample extracts were injected $\left(1 \mu \mathrm{L}\right.$, splitless mode, $250{ }^{\circ} \mathrm{C}$ inlet temperature) into a gas chromatograph (Agilent $6890 \mathrm{~N}$, Agilent Technologies, USA) equipped with a low polarity capillary column (Rxi-5silv, $30 \mathrm{~m} \times 0.25 \mathrm{~mm} \times 0.25 \mu \mathrm{m}$, Restek Co., USA). The GC oven temperature program for GC-O/MS was as follows: $40{ }^{\circ} \mathrm{C}$ $(0.2 \mathrm{~min}) \rightarrow 280^{\circ} \mathrm{C}$ (at $\left.5{ }^{\circ} \mathrm{C} / \mathrm{min}\right) \rightarrow 280{ }^{\circ} \mathrm{C}$ (hold $5 \mathrm{~min}$ ). The carrier gas was helium at $1.35 \mathrm{~mL} / \mathrm{min}$. The GC column outlet was split to both the mass spectrometer and the olfactometry detection port with the flow ratio of 1 to 2 ( 0.45 and $0.9 \mathrm{~mL} / \mathrm{min}$, respectively). The transfer line, mixing chamber, MS source, MS quadrupole and auxiliary column were heated at $150{ }^{\circ} \mathrm{C}, 200{ }^{\circ} \mathrm{C}, 230^{\circ} \mathrm{C}, 150{ }^{\circ} \mathrm{C}$ and $180{ }^{\circ} \mathrm{C}$, respectively. The MS was operated in full-scan mode and solvent delay time was set at $4.5 \mathrm{~min}$.

\subsection{GC $\times$ GC-TOFMS analysis}

A Pegasus-4D GC $\times$ GC-TOFMS system (LECO, USA) equipped with a multi-purpose sampler (Gerstel, Germany) was used for the analysis of the extracts. Comprehensive two-dimensional gas chromatography was linked by a two-stage modulator. The first dimension (the column in the main GC oven) utilized the same capillary column as GC-O/MS with low polarity (Rxi-5silv, $30 \mathrm{~m} \times 0.25 \mathrm{~mm} \times 0.25 \mu \mathrm{m}$, Restek Co., USA), while the second dimension column utilized a polar one (Rxi-17, $1.79 \mathrm{~m} \times 0.10 \mathrm{~mm} \times 0.10 \mu \mathrm{m}$, Restek Co., USA) for better separation of extracts. Liquid nitrogen was filled into a Dewar using a liquid leveler automatically, and was used for cold pulses. Ultrapure helium (He $\geq 99.999 \%$ ) was used as the carrier gas at the constant flow rate of $1 \mathrm{~mL} / \mathrm{min}$. $1 \mu \mathrm{L}$ extracts were introduced using a programmed temperature vaporizing injector in a splitless mode with the inlet temperature of $250{ }^{\circ} \mathrm{C}$. The temperature program of the first column (main GC oven) was optimized as follows: $40{ }^{\circ} \mathrm{C}(0.2 \mathrm{~min}) \rightarrow 280{ }^{\circ} \mathrm{C}$ (at $5{ }^{\circ} \mathrm{C} / \mathrm{min}$ ) $\rightarrow 280{ }^{\circ} \mathrm{C}$ (hold $5 \mathrm{~min}$ ). The temperature of the second oven was programmed from $45{ }^{\circ} \mathrm{C}(0.2 \mathrm{~min})$ to $285^{\circ} \mathrm{C}$ at a rate of $5{ }^{\circ} \mathrm{C} / \mathrm{min}$ with a final hold time of $5 \mathrm{~min}$. The modulator temperature offset was $20{ }^{\circ} \mathrm{C}$ above the first GC oven temperature. The seconddimension separation time (modulation time) was $5.0 \mathrm{~s}$, divided into a hot pulse time of $1.0 \mathrm{~s}$ and a cold pulse time of $1.5 \mathrm{~s}$. The transfer line linking the secondary oven with the mass spectrometer was maintained at $250^{\circ} \mathrm{C}$. The electron energy was $-70 \mathrm{eV}$, and the detector voltage was set at $1575 \mathrm{eV}$. The data acquisition rate was $100 \mathrm{spectra} / \mathrm{s}$, covering a mass range of $50-500 \mathrm{~m} / \mathrm{z}$. The temperature of the ion source was set at $250{ }^{\circ} \mathrm{C}$ (Guo et al., 2015).

\subsection{Odorant identification and confirmation}

Olfactometry blank analysis for GC-O/MS was performed without sample injection to avoid reported odors related to column and septum bleed, and ultrapure water extracted with LLE and solvent blanks was also analyzed by both GC-O/MS and GC $\times$ GC-TOFMS to eliminate odors from impurities in the operating environment and extraction solvents. Only olfactometry peaks with an olfactory detector port score equal to or above 2 were chosen for further identification in GC $\times$ GC-TOFMS. In addition, some water samples were analyzed in duplicate through GC-O/MS to ensure that sensory qualities and intensities characterized by analysts were consistent.

Alkanes (C7-C30,1.0 mg/L) were used for RI calculation to combine olfactometry peaks in GC-O/MS with chromatography peaks in GC $\times$ GC-TOFMS. The RI of an olfactometry peak or chromatography peak was calculated from the retention times (RTs) of the alkanes based on the procedures proposed by van Den Dool (van Den Dool and Dec. Kratz, 1963). The procedure for RI calculation can be found in Supporting Information.

To verify the correspondence between the olfactometry peaks in GC$\mathrm{O} / \mathrm{MS}$ and the corresponding compound information in GC $\times$ GCTOFMS, four typical odorants including IPMP, IBMP, 2-MIB and geosmin were added to the solution of alkanes with a concentration of $50 \mu \mathrm{g} / \mathrm{L} \mathrm{in}$ methylene chloride solvent. Then, full scan mode was employed for analysis of the sample by both GC-O/MS and GC $\times$ GC-TOFMS, of which the mass spectral signals obtained within a 0.2 min window around the olfactometry peaks were isolated and searched against the National Institute of Standards and Technology 2011 (NIST 11, Agilent Chemstation software, USA) mass spectral database for both GC-O/MS and GC $\times$ GC-TOFMS. The search results were prioritized by match probability $(\geq 50 \%)$ and similarity $(\geq 700)$ in GC-O/MS and GC $\times$ GC-TOFMS, respectively. The screening results (the screening procedures are described in Supporting Information) were then compared to previously reported odorants in publicly available commercial odorant databases and publications (Young et al., 1996; Suffet et al., 1999; Watson, 2004; Dunkel et al., 2009). 
For tentatively identified compounds, authentic standards were compared to the olfactometry peaks. Standards with a concentration showing a peak area close to that of the sample were analyzed by GC$\mathrm{O} / \mathrm{MS}$ to verify the olfactometry peaks. Authentic standards with different concentrations were analyzed by GC $\times$ GC-TOFMS in total ion mode, and compared with mass spectra and ion ratio information from the NIST 11 library. The targeted odorants were finally verified by comparing the RI values of the standards analyzed by GC $\times$ GC-TOFMS to the olfactometry peaks from the sample extracts as well as the literature.

\subsection{OAV determination}

Odor activity value (OAV), which was calculated by dividing the odorant concentration by the corresponding OTC, was employed to evaluate the contribution of different odorants to the odor profile (Burdack-Freitag and Schieberle, 2012). Odorants with an OAV equal to or above one are considered to be responsible for odor, and the greater their OAV is, the more they contribute to the odor profile (Pang et al., 2012). In this study, the septic and musty odorants identified were quantified using GC $\times$ GC-TOFMS for all of the six water samples, which were used to calculate the OAVs. The recoveries for the five odorants (2-methylisoborneol, geosmin, dimethyl disulfide, diethyl disulfide, bis(2-chloroisopropyl) ether) were $70.50 \%-90.33 \%$, the details of which can be found in our previous study (Guo et al., 2015).

\section{Results and discussion}

3.1. Effectiveness of combining the data from GC-O/MS and GC $\times$ GC-TOFMS using RIs

As shown in Fig. S1, RTs of the alkanes in GC-O/MS were correlated well $\left(\mathrm{R}^{2}=1.00\right)$ with those in GC $\times$ GC-TOFMS, showing that it was possible to combine the data from the two instruments using RIs. Furthermore, Fig. S2 and Table S1 indicate that good correspondence was displayed for typical odorants (IPMP, IBMP, 2-MIB and geosmin) between chromatography peaks and olfactometry peaks in GC-O/MS, and the RIs for the chromatography peaks of these odorants in $\mathrm{GC} \times \mathrm{GC}$-TOFMS fitted well in the ranges of RIs from the beginning to the end of the olfactometry peaks in GC-O/MS. Thus it is possible to match the olfactometry peaks in GC-O/MS with the chromatography peaks in GC $\times$ GC-TOFMS by using RIs (Eyres et al., 2005; Chin et al., 2011), which will enable us to neglect the compound peaks without odors. Thus many fewer standards will be needed for the confirmation of potential odorants.

\subsection{Selection of pre-concentration method}

Four frequently used pre-concentration methods were compared in terms of their detected olfactometry peak numbers in analyzing the sample of July. As shown in Table 1, a total of 13 olfactometry peaks were obtained using the LLE method, which was much higher than for the other methods including SPME using PDMS/DVB, CAR/PDMS/DVB or CW/PDMS (3-5 peaks), SPE cartridges using C18 or HLB (6-7 peaks), and SDE (7 peaks). Furthermore, all the olfactometry peaks obtained in the other pre-concentration methods could be covered by LLE. Thus LLE was selected for the pre-concentration of odorants.

Three levels of LLE pre-concentration factors $(1000,5000,10,000)$ were then evaluated in GC-O/MS, for which the principle was to distinguish odor signals caused by odorants effectively and suppress background odors during the analysis process. The extracts with preconcentration factors of 5000 and 10,000 generated the same number of olfactometry peaks (13 peaks), which was much higher than that obtained with a pre-concentration factor of 1000 ( 6 peaks). Moreover, the odor intensity smelled for the pre-concentration factor of 5000 was mostly high or very high, while that of 10,000 was too high to bear; therefore, a pre-concentration factor of 5000 was chosen.

\subsection{Odorant identification by GC-O/MS}

FPA evaluation for the Sample of July from the HP River showed that the sample had a musty and septic odor with an intensity of seven and eight, respectively. As shown in Fig. 1 and Table 2, this sample exhibited a complicated olfactometry profile in GC-O/MS. A total of thirteen olfactometry peaks were recorded, with odor descriptors as septic ( 2 peaks), musty ( 1 peak), earthy ( 1 peak), unpleasant ( 2 peaks), fragrant ( 3 peaks), medicinal ( 1 peak), burnt ( 1 peak), cucumber ( 1 peak) and orange (1 peak), respectively. By searching with the NIST spectral library, only two olfactometry peaks (peaks No. 1 and 2) were tentatively assigned to cyclohexanone and diethyl disulfide (DEDS), with a probability of $57.6 \%$ and $90.6 \%$, respectively (Table 2 ), while no compound information could be acquired for the other eleven olfactometry peaks in GC-O/MS. Similar results were also reported in analyzing samples from wine, food and other industries using GC-O analysis, mainly due to the low instrument detection limit or co-elution phenomenon for one-dimensional GC systems (Dalluge et al., 2003; Chin et al., 2011).

Table 1

Comparison of extraction efficiencies using different pre-concentration methods.

\begin{tabular}{|c|c|c|c|c|c|c|c|c|}
\hline \multicolumn{2}{|c|}{ Olfactometry peaks } & \multicolumn{7}{|c|}{ Pre-concentration method } \\
\hline \multirow[t]{2}{*}{ Descriptor } & \multirow[t]{2}{*}{ RT (min) } & \multirow[t]{2}{*}{ SDE } & \multicolumn{3}{|l|}{ SPME } & \multicolumn{2}{|l|}{ SPE } & \multirow[t]{2}{*}{$\operatorname{LLE}^{c}$} \\
\hline & & & PDMS/DVB & CW/PDMS & CAR/PDMS/DVB & HLB & $\mathrm{C} 18$ & \\
\hline Unpleasant & $7.55-7.67$ & $-{ }^{a}$ & - & + & + & + & - & + \\
\hline Septic & $8.36-8.49$ & - & - & - & - & + & + & + \\
\hline Burnt & $9.17-9.30$ & $++^{\mathrm{b}}$ & - & - & - & - & + & + \\
\hline Fragrant & $9.73-9.88$ & + & + & + & + & + & + & + \\
\hline Septic & $11.70-11.83$ & + & - & - & + & + & + & + \\
\hline Unpleasant & $13.88-14.01$ & + & - & + & - & - & - & + \\
\hline Cucumber & $14.58-14.70$ & - & - & - & - & + & - & + \\
\hline Medicinal & $15.26-15.38$ & - & + & - & - & - & + & + \\
\hline Musty & $15.80-15.91$ & + & + & + & + & + & + & + \\
\hline Orange & $16.16-16.27$ & - & - & + & - & + & - & + \\
\hline Fragrant & $18.87-18.98$ & + & - & - & - & - & - & + \\
\hline Earthy & $21.76-21.87$ & + & - & - & - & - & - & + \\
\hline Fragrant & $22.85-22.97$ & - & - & - & - & - & - & + \\
\hline \multicolumn{2}{|c|}{ Total olfactometry peaks } & 7 & 3 & 5 & 4 & 7 & 6 & 13 \\
\hline
\end{tabular}

\footnotetext{
a No olfactometry peak was detected

b Olfactometry peak was detected.

c Concentration factor: 5000 .
} 


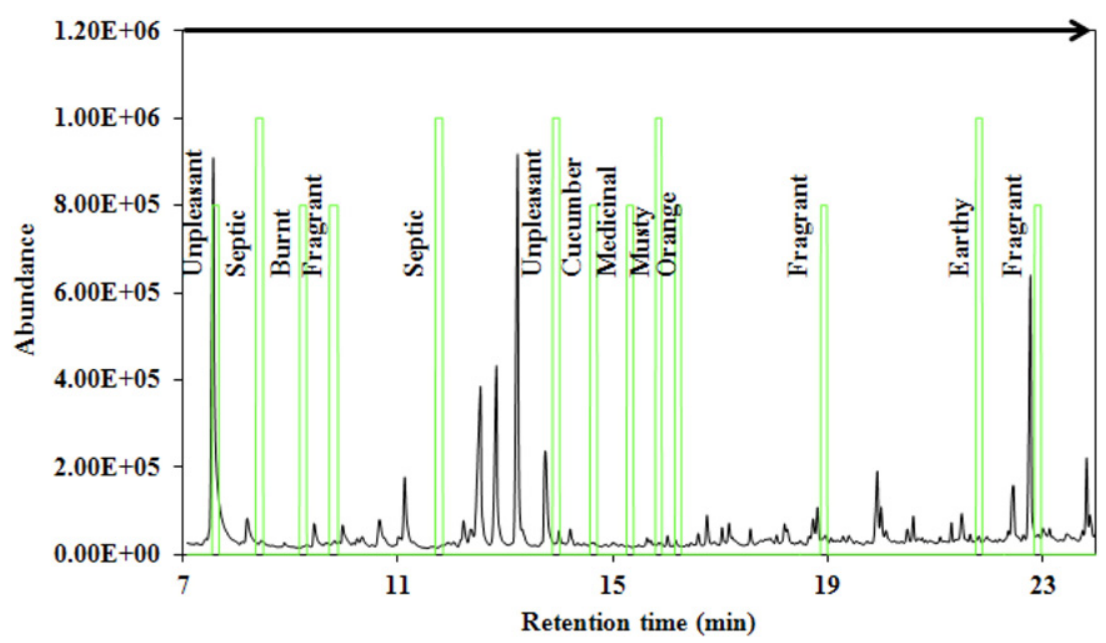

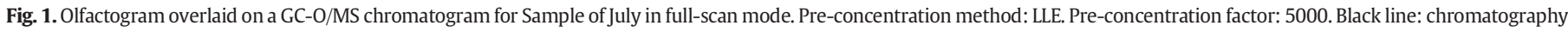
peaks. Green line: olfactometry peaks.

3.4. Odorant identification by combining the data from GC-O/MS and GC $\times$ GC-TOFMS using RIs

Fig. 2 shows the GC $\times$ GC-TOFMS analysis results for the same sample. In order to find the compound corresponding to an olfactometry peak in GC-O/MS, the candidate compounds with similar RI values were screened first based on the results of a spectral library search of compounds having a similarity above 700 (Dalluge et al., 2003), and then confirmed by authentic standards, which were selected based on their reported odor characteristics, RI values and mass spectral ion information.

It was found that GC $\times$ GC-TOFMS could generate many more chromatography peaks (2858) than GC-O/MS (486). As shown in Fig. 2, the group-type separation of homologous compounds could be recognized clearly for most of the alkanes (Adahchour et al., 2008). Small alkanes such as C7, C8 and C9 were close to column bleedings, and need to be identified carefully. Heavy tailing for larger alkanes, such as octadecane, eicosane, docosane, etc., emerged in the second dimension of GC $\times$ GCTOFMS. Moreover, C29 and C30 could not be detected. However, such phenomena did not affect the results of RI calculation. On the other hand, three saturated aldehydes including heptanal, decanal, undecanal did not display a group-type phenomenon, which might be attributed to wrap-around because of the long second dimension column (Adahchour et al., 2008).

Over thirty potential odorants corresponding to the thirteen olfactometry peaks in the Sample of July were tentatively identified by GC $\times$ GC-TOFMS with high similarity matching (above 700) to compounds in the NIST 11 MS library, as shown in Table 2. More potential odorants were identified by combining the data from GC-O/MS and GC $\times$ GC-TOFMS using RIs. Finally, thirteen odorants were confirmed through authentic standards. On the other hand, another septic odorant dimethyl disulfide (DMDS), whose olfactometry peak was not detected due to solvent delay, was detected by GC $\times$ GC-TOFMS analysis. So a total of fourteen odorants were confirmed in the Huangpu River sample.

As shown in Table 2, the septic odor for peaks No. 2 and 5 could be assigned to DEDS and bis(2-chloroisopropyl) ether, respectively. Including DMDS, three septic odorants were detected in the sample. The sulfur-containing compounds such as DEDS, DMDS, DMTS with OTC values as low as 20,30 , and $10 \mathrm{ng} / \mathrm{L}$ have been frequently reported as typical odorants in source water (Watson, 2004; Yang et al., 2008). DMTS was detected with a concentration as high as $11,399 \mathrm{ng} / \mathrm{L}$ in the Wuxi odor incident (Yang et al., 2008), and much lower concentrations in HP River source water (Yu et al., 2009). However, DMTS was not detected in this study. DMTS has been reported as the major odorant identified from algal cultures or algae-induced black blooms (Watson, 2004), while DEDS and DMDS were more associated with bio-industry (Hwang et al., 1995; Smet and Van Langenhove, 1998), swine operation processes (Schiffman et al., 2001) and polluted rivers (Sheng et al., 2008). The cyanobacterial blooms in Taihu Lake, the major water source of the HP River, have been improved due to extensive water pollution control efforts in the region (Xu et al., 2010), which might be a part of the reason for the disappearance of DMTS. Bis(2-chloroisopropyl) ether, as a septic odorant, has been discovered in drinking water contaminated by industrial outfall (Kleopfer and Fairless, 1972; Steltenpohl and Graczova, 2008). Its OTC was evaluated to be $17 \mathrm{ng} / \mathrm{L}$ by the 3-alternative forced choice (3-AFC) method (ASTM, 1997), as shown in Table 3. This odorant was also detected in different source waters of China, including the Huangpu River source water, in our previous investigation (Yu et al., 2009).

Olfactometry peaks No. 3 and 8 were assigned to $\alpha$-methyl-styrene and 2,6-dichloro-phenol, respectively. The former was reported to have an irritant odor (Schiffman et al., 2001) but was rarely reported in drinking water, while the latter was reported to have a medicinal odor with an OTC as low as $20 \mathrm{ng} / \mathrm{L}$. 2,6-dichloro-phenol and some other halophenols are often implicated in medicinal odors in drinking water (Young et al., 1996; Agus et al., 2012). The presence of diverse chemicals, including bis(2-chloroisopropyl) ether, $\alpha$-methyl-styrene, 2,6-dichloro-phenol, DEDS and DMDS, suggested that the river source water is suffering from different types of domestic and industrial pollution. Actually, this river is located in the most populated and industrialized area in China, which makes it easily contaminated (Ren et al., 2003; Yang et al., 2007; Wang et al., 2008), and further protection measures should be taken in the future.

The musty and earthy odors at olfactometry peaks No. 9 and 12 could be assigned to 2-MIB and geosmin, respectively. 2-MIB has been found to be the major odorant for musty odor in the HP River produced by Phormidium spp. in our previous study (Li et al., 2012; Sun et al., 2013). However, geosmin was detected as the earthy odorant in this study, which might be attributed to algal species transformation in recent years, which needs further research efforts. Other algal metabolites (Yasuhara and Fuwa, 1982; Watson, 2004; Zhang et al., 2013), including heptanal, 2-nonenal, 2,6-nonadienal, decanal, undecanal, butyl butanoate and $\beta$-ionone, were identified as associated with the remaining olfactometry peaks. Heptanal could produce a fishy/oily odor with an OTC of $3000 \mathrm{ng} / \mathrm{L}$, and 2-nonenal and 2,6-nonadienal could produce a cucumber odor with OTCs of $800 \mathrm{ng} / \mathrm{L}$ and $80 \mathrm{ng} / \mathrm{L}$, respectively 
Table 2

Summary of characteristic olfactometry peaks and identified odorants for Sample of July.

\begin{tabular}{|c|c|c|c|c|c|c|c|c|c|}
\hline \multirow{2}{*}{$\begin{array}{l}\text { Peak } \\
\text { no. }\end{array}$} & \multicolumn{2}{|c|}{ Olfactometry peaks } & \multirow[t]{2}{*}{ Potential odorants in GC-O/MS } & \multirow[t]{2}{*}{ Probability in GC-O } & \multirow{2}{*}{$\begin{array}{l}\text { Potential odorants in GC } \times \\
\text { GC-TOFMS }\end{array}$} & \multirow[t]{2}{*}{ Similarity in GC $\times$ GC } & \multirow[t]{2}{*}{ Confirmed odorants } & \multirow[t]{2}{*}{ RI } & \multirow[t]{2}{*}{ Descriptor } \\
\hline & Descriptor & RI & & & & & & & \\
\hline - & Septic & $355(\mathrm{~s})$ & - & - & Dimethyl disulfide & 926 & Dimethyl disulfide & 743 & Septic \\
\hline \multirow[t]{3}{*}{1} & Unpleasant & $902-908$ & Cyclohexanone & $57.6 \%$ & Heptanal & 926 & Heptanal & 905 & Fishy, unpleasant \\
\hline & & & & & Cyclohexanone & 822 & & & \\
\hline & & & & & 2-Ethyl-pyridine & 883 & & & \\
\hline \multirow[t]{4}{*}{2} & Septic & 931-939 & Diethyl disulfide & $90.6 \%$ & Diethyl disulfide & 937 & Diethyl disulfide & 932 & Septic, rancid \\
\hline & & & & & 2,4-Dimethyl-pyridine & 922 & & & \\
\hline & & & & & 2-Chloro-pyridine & 925 & & & \\
\hline & & & & & 2-Cyclohexen-1-one & 840 & & & \\
\hline \multirow[t]{3}{*}{3} & Burnt & 960-966 & Tetrahydro-2,2,4,4-tetramethyl-Furan & $<50 \%$ & $\alpha$-Methyl-styrene & 920 & $\alpha$-Methyl-styrene & 962 & Irritant, burnt \\
\hline & & & 2,5-Dimethyl-2,5-Hexanediol & & 3-Ethyl-pyridine & 847 & & & \\
\hline & & & 7-Tridecanone & & 4-Ethyl-octene & 778 & & & \\
\hline \multirow[t]{4}{*}{4} & Fragrant & $978-986$ & 2-Cyclohexylpiperidine & $<50 \%$ & Butyl butanoate & 904 & Butyl butanoate & 981 & Flower, fragrant \\
\hline & & & Glutaconic acid & & 1-Octen-3-one & 811 & & & \\
\hline & & & & & 1-Octen-3-ol & 784 & & & \\
\hline & & & & & Aniline & 938 & & & \\
\hline \multirow[t]{3}{*}{5} & Septic & $1048-1052$ & 1,8-Nonadien-3-ol & $<50 \%$ & Bis(2-chloroisopropyl) ether & 914 & Bis(2-chloroisopropyl) ether & 1049 & Septic, pesticide \\
\hline & & & Carveol & & 5-Undecene & 800 & & & \\
\hline & & & & & 2-Methyl-phenol & 868 & & & \\
\hline \multirow[t]{3}{*}{6} & Unpleasant & $1123-1128$ & 4-Ethyl-benzenamine & $<50 \%$ & 2-Nonenal & 932 & 2-Nonenal & 1127 & Irritant, unpleasant \\
\hline & & & $\mathrm{N}$-ethyl-benzenamine & & o-Cymene & 912 & & & \\
\hline & & & $\begin{array}{l}\text { 5-Ethyl-2-methyl-pyridine } \\
\text { 4-Isopropylpyridine }\end{array}$ & & Isophorone & 734 & & & \\
\hline \multirow[t]{2}{*}{7} & Cucumber & $1152-1155$ & 3,6-Dimethyl-octan-2-one & $<50 \%$ & 2,6-Nonadienal & 902 & 2,6-Nonadienal & 1155 & Cucumber \\
\hline & & & 5-Octadecenal & & 2,1,3-Benzothiadiazole & 894 & & & \\
\hline \multirow[t]{3}{*}{8} & Medicinal & $1173-1175$ & Tridecanedial & $<50 \%$ & 2-Decen-1-ol & 849 & 2,6-Dichloro-phenol & 1173 & Medicinal, phenolic \\
\hline & & & 3,6-Dimethyl-octan-2-one & & 2,6-Dichloro-phenol & 761 & & & \\
\hline & & & 5-Octadecenal & & Ethyl benzoate & 817 & & & \\
\hline \multirow[t]{2}{*}{9} & Musty & $1190-1196$ & Folic Acid & $<50 \%$ & 2-Decanone & 883 & 2-Methylisoborneol & 1192 & Musty, moldy \\
\hline & & & $\begin{array}{l}\text { 3,6-Dimethyl-octan-2-one } \\
\text { 5-Octadecenal }\end{array}$ & & 2-Methylisoborneol & 804 & & & \\
\hline \multirow[t]{2}{*}{10} & Orange & $1205-1210$ & 3-Hydroxy-dodecanoic acid & $<50 \%$ & Decanal & 919 & Decanal & 1206 & Orange flavor \\
\hline & & & $\begin{array}{l}\text { 2-Decen-1-ol } \\
\text { trans-2-Undecenoic acid }\end{array}$ & & 3-Phenyl-2-propenal & 776 & & & \\
\hline \multirow[t]{2}{*}{11} & Fragrant & 1305-1309 & 3,6-Dimethyl-octan-2-one & $<50 \%$ & Undecanal & 914 & Undecanal & 1306 & Fragrant \\
\hline & & & $\begin{array}{l}\text { trans-2-Hexadecenoic acid } \\
\text { 4-Octadecenal }\end{array}$ & & 2-Methyl-quinoxaline & 756 & & & \\
\hline \multirow[t]{2}{*}{12} & Earthy & $1419-1423$ & 1,3-Dimethyl-naphthalene & $<50 \%$ & Geosmin & 833 & Geosmin & 1420 & Earthy, musty \\
\hline & & & 3,6-Dimethyl-octan-2-one & & 1,7-Dimethyl-naphthalene & 726 & & & \\
\hline \multirow[t]{2}{*}{13} & Fragrant & $1468-1472$ & Geranylisovalerate & $<50 \%$ & Ionone & 895 & Ionone & 1470 & Fragrant \\
\hline & & & 2-Hexadecanol & & 2-Phenyl-pyridine & 755 & & & \\
\hline
\end{tabular}




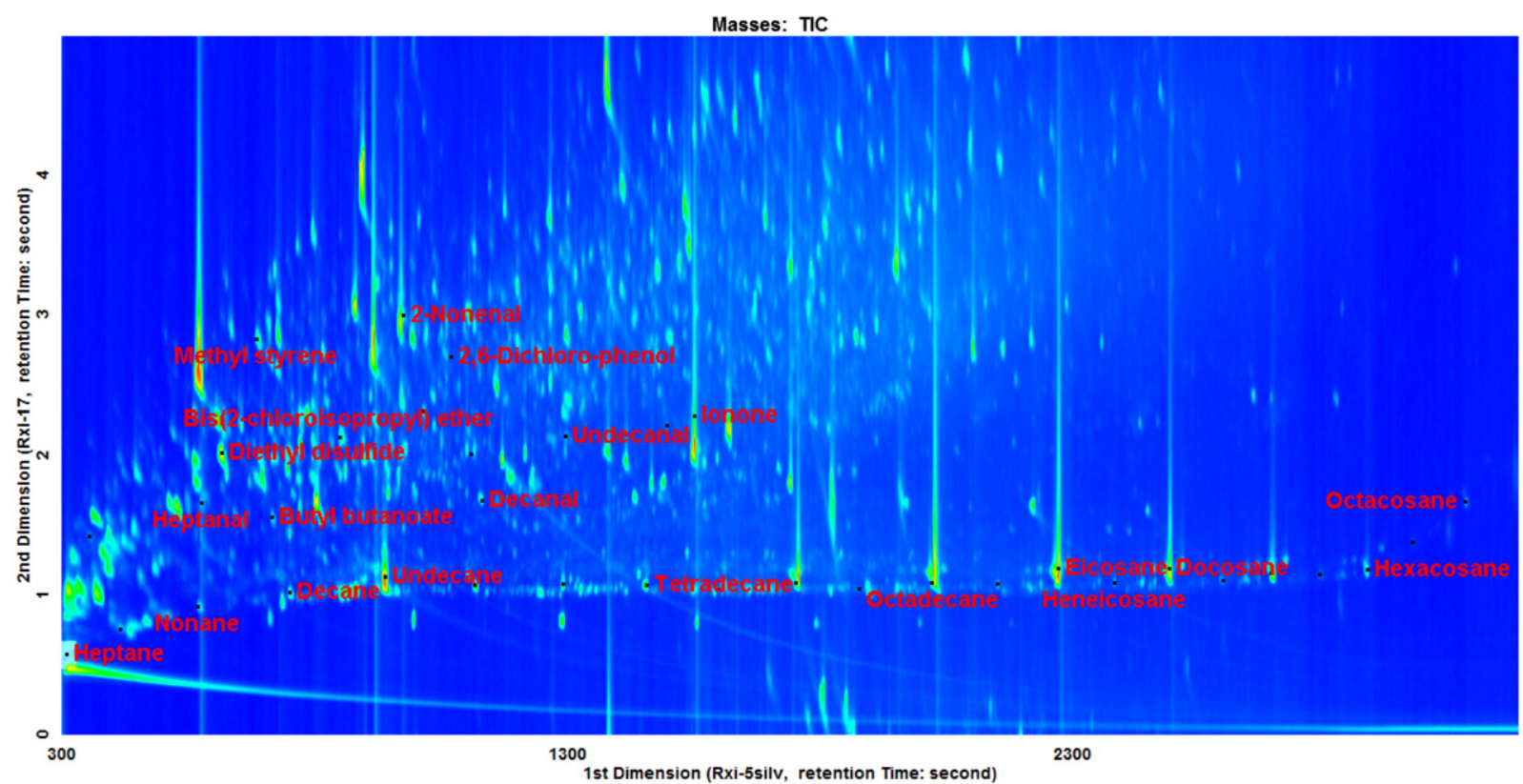

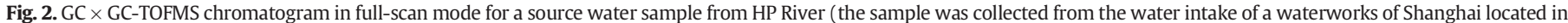
the lower HP River on 15th July 2014)

(Watson, 2004). Decanal, as a fruity/orange-like odorant, has been detected in drinking water (Bao et al., 1997; Suffet et al., 2004). Undecanal and $\beta$-ionone have been reported as typical compounds in algal metabolism processes (Friedrich and Acree, 1998; Watson, 2004), while butyl butanoate was reported as an important flavor compound in fruit and wine industries (Welke et al., 2012; Pino and Febles, 2013). The presence of many algal metabolites should be associated with Taihu Lake, which is located in the upstream of the river. All the identified odorants' characteristics are shown in Table 3 . Thus the method, combining the data from GC-O/MS and GC $\times$ GC-TOFMS using RIs, was used for the identification of potential odorants in Huangpu River source water successfully, which allowed the assignment of potential odorants in GC $\times$ GC-TOFMS to olfactometry peaks detected by GC-O/MS.

\subsection{Evaluation of the contributions of odorants to the septic odor using OAV}

Table S2 shows the identification results for the 6 HP River source water samples taken from July to December 2014 obtained by combining the data from GC-O/MS and GC $\times$ GC-TOFMS using RIs. Similar to our previous study (Sun et al., 2013), 2-MIB and geosmin were detected in all of the samples. At the same time, the three septic odorants bis(2chloroisopropyl) ether, DEDS and DMDS were always detected, while some other odorants were occasionally detected.

There were two typical odors characterized as septic (intensities: 4-8) and musty (intensities: 4-7) according to the FPA results (Table 4), showing that the source water was associated with constant septic and musty odors. The three septic odorants, bis(2-chloroisopropyl) ether, DEDS and DMDS, and the two musty odorants in the 6 samples were quantified, as shown in Table S3. Bis(2-chloroisopropyl) ether, DEDS and DMDS varied in concentration ranges between 31.24 and 54.34, 31.28-39.16 and 10.97-72.52 ng/L, respectively, and geosmin and 2-MIB were 17.49-45.74 and 9.58-18.86 ng/L. OAVs for the musty and septic odorants were calculated using the quantification results in Table S3. As shown in Table 4, for the musty odor, geosmin and 2-MIB exhibited a constant OAV above one (OAVs: 4.37-11.44 and 1.131.89 , respectively). A compound with an OAV equal to or above 1.0 is considered to be important in constituting the odor profile (BurdackFreitag and Schieberle, 2012). Thus it was clear that the musty odor was mainly contributed by the two algal metabolites, particularly geosmin, considering its much higher OAV. This result was reasonable since these two compounds have been reported to be responsible for the musty odor for many odor episodes in the world (Suffet et al., 1999; Lin et al., 2002).

On the other hand, bis(2-chloroisopropyl) ether and DEDS always exhibited an OAV value above 1.0 (OAVs: $1.84-3.05$ and 1.56-1.96, respectively), while the OAV for DMDS varied between 0.37 and 2.42 . So it was clear that the above three odorants may have played an important role in constituting the septic odor (Benkwitz et al., 2012).

Since the source water was constantly contaminated with the musty and septic odorants, the interference between the two groups was evaluated using some simple tests (Whelton and Dietrich, 2004), as shown in Fig. S3. It was found that the septic odor caused by DMDS and DEDS at a relatively high concentration could suppress the musty odor caused by 2-MIB, while the addition of 2-MIB in DMDS and DEDS solutions could strengthen the septic odor intensities, particularly at lower DMDS or DEDS concentrations. The relationship between OAV and FPA for 2MIB and geosmin in ultrapure water was compared with that between the sum of the two musty odorants' OAV and FPA values in the Sample of July as shown in Fig. S4. It was clear that the result acquired in ultrapure water was similar to that in the source water sample, showing that geosmin and 2-MIB should be the major odorants responsible for the musty odor. The suppression of musty odor by DMDS and DEDS might not be so significant, because of the low concentrations of the septic odorants. However, for the septic odor, a significant difference could be observed in the results between the ultrapure water and the source water sample. The three septic odorants alone could not explain the FPA results of the source water. There may be some unknown septic odorants since the septic odor profile is very complicated (Suffet et al., 1999). At the same time, the source water contained diverse odorants as described above, which could also strengthen the septic odor, like 2-MIB. Therefore, additional studies including aroma extract dilution analysis (AEDA), reconstitution and omission tests, etc. (Benkwitz et al., 2012) are necessary to clarify the whole odor profile in the HP River. 
Table 3

Odorants characteristics.

\begin{tabular}{|c|c|c|c|c|}
\hline Odorants & Target ions, $\mathrm{m} / \mathrm{z}$ & OTC (ng/L) & CAS & Structure \\
\hline Dimethyl disulfide & $94,79,45$ & $30^{\mathrm{a}}$ & $624-92-0$ & \\
\hline Heptanal & $70,41,44$ & $3000^{\mathrm{b}}$ & $111-71-7$ & \\
\hline Diethyl disulfide & $122,66,29$ & $20^{\mathrm{a}}$ & $110-81-6$ & \\
\hline$\alpha$-Methyl-styrene & $118,117,103$ & n.a. ${ }^{\mathrm{c}}$ & $98-83-9$ & \\
\hline Butyl butanoate & $71,43,56$ & n.a. & $109-21-7$ & \\
\hline Bis(2-chloroisopropyl) ether & $45,41,121$ & $17^{\mathrm{a}}$ & $39638-32-9$ & \\
\hline 2-Nonenal & $41,43,29$ & $25^{\mathrm{a}}$ & $2463-53-8$ & \\
\hline 2,3-Dimethyl-phenol & $107,122,77$ & n.a. & $526-75-0$ & \\
\hline 2,6-Nonadienal & $41,69,70$ & $80^{\mathrm{b}}$ & $17587-33-6$ & \\
\hline 2,6-Dichloro-phenol & $162,164,63$ & $20^{\mathrm{b}}$ & $87-65-0$ & \\
\hline 2-Methylisoborneol & $95,107,108$ & $10^{\mathrm{b}}$ & $2371-42-8$ & \\
\hline Decanal & $29,43,41$ & n.a. & $112-31-2$ & \\
\hline Undecanal & $41,43,57$ & n.a. & $112-44-7$ & \\
\hline Geosmin & $112,111,125$ & $4^{\mathrm{b}}$ & $19700-21-1$ & \\
\hline Ionone & $121,43,93$ & $7^{\mathrm{b}}$ & 8013-90-9 & \\
\hline
\end{tabular}

a OTC was determined by 3-AFC.

b OTC was reported in reference.

c OTC was unreported in reference and not determined.

\section{Conclusions}

In this study, the potential septic odorants in HP River source water were identified successfully by a method combining the data from GC$\mathrm{O} / \mathrm{MS}$ and GC $\times$ GC-TOFMS using RIs. This method allowed the assignment of potential odorants to all 13 olfactometry peaks (odorants identification was ineffective in GC-O/MS) detected by GC-O/MS for a
HP River source water sample, which indicated that odorants could be identified effectively in highly complex water samples by combining the data from GC-O/MS and GC $\times$ GC-TOFMS using RIs. OAV evaluation results for 6 samples over a period of a half year indicated that the musty odor was probably caused by geosmin and 2-MIB, while the septic odor may be mainly related to bis(2-chloroisopropyl) ether, DEDS and DMDS. There might be some unknown septic odorants in the source

Table 4

OAV calculation of septic and musty odorants in HP River from July to December 2014.

\begin{tabular}{|c|c|c|c|c|c|c|c|}
\hline \multirow[t]{2}{*}{ Odor type } & \multirow[t]{2}{*}{ Odorants } & \multirow{2}{*}{$\begin{array}{l}\text { July } \\
\text { Swampy (eight), musty } \\
\text { (seven) }\end{array}$} & \multirow{2}{*}{$\begin{array}{l}\text { August } \\
\text { Swampy (seven), } \\
\text { musty (four) }\end{array}$} & \multirow{2}{*}{$\begin{array}{l}\text { September } \\
\text { Swampy (four), } \\
\text { musty (five) }\end{array}$} & \multirow{2}{*}{$\begin{array}{l}\text { October } \\
\text { Swampy (five), } \\
\text { musty (five) }\end{array}$} & \multirow{2}{*}{$\begin{array}{l}\frac{\text { November }}{\text { Swampy (five), }} \\
\text { musty (five) }\end{array}$} & \multirow{2}{*}{$\begin{array}{l}\text { December } \\
\text { Swampy (five) } \\
\text { musty (five) }\end{array}$} \\
\hline & & & & & & & \\
\hline \multirow[t]{3}{*}{ Septic } & Dimethyl disulfide & 2.42 & 0.77 & 0.53 & 0.37 & 0.52 & 0.65 \\
\hline & Diethyl disulfide & 1.83 & 1.80 & 1.68 & 1.56 & 1.96 & 1.78 \\
\hline & Bis (2-chloroisopropyl) ether & 3.05 & 3.20 & 2.22 & 2.15 & 1.84 & 2.19 \\
\hline \multirow[t]{2}{*}{ Musty } & 2-Methylisoborneol & 1.13 & 1.20 & 1.30 & 1.13 & 1.35 & 1.89 \\
\hline & Geosmin & 11.44 & 4.37 & 5.30 & 4.88 & 5.28 & 5.43 \\
\hline
\end{tabular}


water. Other identified odorants may have also contributed to the septic odor of the HP River. The synergistic or masking effects among different odorants need to be explored in the future.

\section{Acknowledgements}

This study was supported by funds from the National Natural Science Foundation of China (No. 21377144), Major Science and Technology Program for Water Pollution Control and Treatment (No. 2015ZX07406001, No. 2012ZX07403-002), and the "135" Major Project of the Research Center for Eco-Environmental Science (YSW2013A02).

\section{Appendix A. Supplementary data}

Supplementary data to this article can be found online at http://dx. doi.org/10.1016/j.scitotenv.2016.03.003.

\section{References}

Adahchour, M., van Stee, L.L.P., Beens, J., Vreuls, R.J.J., Batenburg, M.A., Brinkman, U.A.T., 2003. Comprehensive two-dimensional gas chromatography with time-of-flight mass spectrometric detection for the trace analysis of flavour compounds in food. J. Chromatogr. A 1019 (1-2), 157-172.

Adahchour, M., Beens, J., Brinkman, U.A.T., 2008. Recent developments in the application of comprehensive two-dimensional gas chromatography. J. Chromatogr. A 1186 (12), 67-108.

Agus, E., Zhang, L.F., Sedlak, D.L., 2012. A framework for identifying characteristic odor compounds in municipal wastewater effluent. Water Res. 46 (18), 5970-5980.

APHA, 2005. Standard Methods for the Examination of Water and Wastewater. APHA American Public Health Association.

ASTM, 1997. Standard Practice Designation E 679-91. American Society for Testing and Materials, pp. 34-38.

Bao, M.L., Barbieri, K., Burrini, D., Griffini, O., Pantani, F., 1997. Determination of trace levels of taste and odor compounds in water by microextraction and gas chromatographyion-trap detection-mass spectrometry. Water Res. 31 (7), 1719-1727.

Benkwitz, F., Nicolau, L., Lund, C., Beresford, M., Wohlers, M., Kilmartin, P.A., 2012. Evaluation of key odorants in sauvignon blanc wines using three different methodologies. J. Agric. Food Chem. 60 (25), 6293-6302.

Burdack-Freitag, A., Schieberle, P., 2012. Characterization of the key odorants in raw Italian hazelnuts (Corylus avellana L. var. Tonda Romana) and roasted hazelnut paste by means of molecular sensory science. J. Agric. Food Chem. 60 (20), 5057-5064.

Chen, J., Xie, P., Ma, Z., Niu, Y., Tao, M., Deng, X., Wang, Q., 2010. A systematic study on spatial and seasonal patterns of eight taste and odor compounds with relation to various biotic and abiotic parameters in Gonghu Bay of Lake Taihu, China. Sci. Total Environ. 409 (2), 314-325.

Chin, S.T., Eyres, G.T., Marriott, P.J., 2011. Identification of potent odourants in wine and brewed coffee using gas chromatography-olfactometry and comprehensive twodimensional gas chromatography. J. Chromatogr. A 1218 (42), 7487-7498.

d'Acampora Zellner, B., Dugo, P., Dugo, G., Mondello, L., 2008. Gas chromatographyolfactometry in food flavour analysis. J. Chromatogr. A 1186 (1-2), 123-143.

Dalluge, J., Beens, J., Brinkman, U.A.T., 2003. Comprehensive two-dimensional gas chromatography: a powerful and versatile analytical tool. J. Chromatogr. A 1000 (1-2), 69-108.

Davi, M.L., Gnudi, F., 1999. Phenolic compounds in surface water. Water Res. 33 (14), 3213-3219.

Dunkel, M., Schmidt, U., Struck, S., Berger, L., Gruening, B., Hossbach, J., Jaeger, I.S., Effmert, U., Piechulla, B., Eriksson, R., Knudsen, J., Preissner, R., 2009. Nucleic acids research. Nucleic Acids Res. 37, D291-D294.

Eyres, G., Dufour, J.P., Hallifax, G., Sotheeswaran, S., Marriott, P.J., 2005. Identification of character-impact odorants in coriander and wild coriander leaves using gas chromatography-olfactometry (GCO) and comprehensive two-dimensional gas chromatography-time-of-flight mass spectrometry (GC $\times$ GC-TOFMS). J. Sep. Sci. 28 (9-10), 1061-1074.

Friedrich, J.E., Acree, T.E., 1998. Gas chromatography olfactometry (GC/O) of dairy products. Int. Dairy J. 8 (3), 235-241.

Guo, Q.Y., Li, X., Yu, J.W., Zhang, H.F., Zhang, Y., Yang, M., Lu, N., Zhang, D., 2015. Comprehensive two-dimensional gas chromatography with time-of-flight mass spectrometry for the screening of potent swampy/septic odor-causing compounds in two drinking water sources in China. Anal. Methods 7 (6), 2458-2468.

Hwang, Y., Matsuo, T., Hanaki, K., Suzuki, N., 1995. Identification and quantification of sulfur and nitrogen-containing odorous compounds in waste water. Water Res. 29 (2), 711-718.

Kleopfer, R.D., Fairless, B.J., 1972. Characterization of organic components in a municipal water supply. Environ. Sci. Technol. 6 (12), 1036-\&.
Li, Z.L., Hobson, P., An, W., Burch, M.D., House, J., Yang, M., 2012. Earthy odor compounds production and loss in three cyanobacterial cultures. Water Res. 46 (16), 5165-5173.

Lin, T.F., Wong, J.Y., Kao, H.P., 2002. Correlation of musty odor and 2-MIB in two drinking water treatment plants in South Taiwan. Sci. Total Environ. 289 (1-3), 225-235.

Pandey, R.A., Padoley, K.V., Mukherji, S.S., Mudliar, S.N., Vaidya, A.N., Rajvaidya, A.S. Subbarao, T.V., 2007. Biotreatment of waste gas containing pyridine in a biofilter. Bioresour. Technol. 98 (12), 2258-2267.

Pang, X.L., Guo, X.F., Qin, Z.H., Yao, Y.B., Hu, X.S., Wu, J.H., 2012. Identification of aromaactive compounds in Jiashi muskmelon juice by GC-O-MS and OAV calculation. J. Agric. Food Chem. 60 (17), 4179-4185.

Pino, J.A., Febles, Y., 2013. Odour-active compounds in banana fruit cv. giant cavendish. Food Chem. 141 (2), 795-801.

Ren, W., Zhong, Y., Meligrana, J., Anderson, B., Watt, W.E., Chen, J., Leung, H.L., 2003. Urbanization, land use, and water quality in Shanghai: 1947-1996. Environ. Int. 29 (5), 649-659.

Schiffman, S.S., Bennett, J.L., Raymer, J.H., 2001. Quantification of odors and odorants from swine operations in North Carolina. Agric. For. Meteorol. 108 (3), 213-240.

Sheng, Y., Chen, F., Yu, Y., Wang, X., Sheng, G., Fu, J., Zeng, E.Y., 2008. Emission of volatile organic sulfur compounds from a heavily polluted river in Guangzhou, South China. Environ. Monit. Assess. 143 (1-3), 121-130.

Smet, E., Van Langenhove, H., 1998. Abatement of volatile organic sulfur compounds in odorous emissions from the bio-industry. Biodegradation 9 (3-4), 273-284.

Steltenpohl, P., Graczova, E., 2008. Vapor-liquid equilibria of selected components in propylene oxide production. J. Chem. Eng. Data 53 (7), 1579-1582.

Suffet, I.H., Khiari, D., Bruchet, A., 1999. The drinking water taste and odor wheel for the millennium: beyond geosmin and 2-methylisoborneol. Water Sci. Technol. 40 (6), $1-13$.

Suffet, I.M., Schweitze, L., Khiari, D., 2004. Olfactory and chemical analysis of taste and odor episodes in drinking water supplies. Rev. Environ. Sci. Biotechnol. 3 (1), 3-13.

Sun, D.L., Yu, J.W., An, W., Yang, M., Chen, G.G., Zhang, S.J., 2013. Identification of causative compounds and microorganisms for musty odor occurrence in the Huangpu River China. J. Environ. Sci. 25 (3), 460-465.

Tomar, M., Abdullah, T.H.A., 1994. Evaluation of chemicals to control the generation of malodorous hydrogen-sulfide in waste-water. Water Res. 28 (12), 2545-2552.

van Den Dool, H., Dec. Kratz, P., 1963. A generalization of the retention index system including linear temperature programmed gas-liquid partition chromatography. J. Chromatogr. A 11, 463-471.

van Ruth, S.M., 2001. Methods for gas chromatography-olfactometry: a review. Biomol Eng. 17 (4-5), 121-128.

Vaughn, J.C., 1967. Tastes and odors in water supplies. Environ. Sci. Technol. 1 (9), 703-709.

Wang, J., Da, L., Song, K., Li, B.L., 2008. Temporal variations of surface water quality in urban, suburban and rural areas during rapid urbanization in Shanghai, China. Environ. Pollut. 152 (2), 387-393.

Wang, Y., Yu, J., Zhang, D., Yang, M., 2014. Addition of hydrogen peroxide for the simultaneous control of bromate and odor during advanced drinking water treatment using ozone. J. Environ. Sci. 26 (3), 550-554.

Watson, S.B., 2004. Aquatic taste and odor: a primary signal of drinking - water integrity. J. Toxic. Environ. Health A 67 (20-22), 1779-1795.

Watson, S.B., Brownlee, B., Satchwill, T., McCauley, E., 1999. The use of solid phase microextraction (SPME) to monitor for major organoleptic compounds produced by chrysophytes in surface waters. Water Sci. Technol. 40 (6), 251-256.

Welke, J.E., Manfroi, V., Zanus, M., Lazarotto, M., Zini, C.A., 2012. Characterization of the volatile profile of Brazilian merlot wines through comprehensive two dimensional gas chromatography time-of-flight mass spectrometric detection. J. Chromatogr. A 1226, 124-139.

Whelton, A.J., Dietrich, A.M., 2004. Relationship between intensity, concentration, and temperature for drinking water odorants. Water Res. 38 (6), 1604-1614.

Wright, E., Daurie, H., Gagnon, G.A., 2014. Development and validation of an SPE-GC-MS/ MS taste and odour method for analysis in surface water. Int. J. Environ. Anal. Chem. 94 (13), 1302-1316.

Xu, H., Paerl, H.W., Qin, B., Zhu, G., Gaoa, G., 2010. Nitrogen and phosphorus inputs control phytoplankton growth in eutrophic Lake Taihu, China. Limmol. Oceanogr. 55 (1) 420-432.

Yang, H.J., Shen, Z.M., Zhang, J.P., Wang, W.H., 2007. Water quality characteristics along the course of the Huangpu River (China). J. Environ. Sci. 19 (10), 1193-1198.

Yang, M., Yu, J.W., Li, Z.L., Guo, Z.H., Burch, M., Lin, T.F., 2008. Taihu Lake not to blame for Wuxi's woes. Science 319 (5860), 158

Yasuhara, A., Fuwa, K., 1982. Characterization of odorous compounds in rotten blue-green algae. Agric. Biol. Chem. 46 (7), 1761-1766.

Young, C., Suffet, I.H., 1999. Development of a standard method - analysis of compounds causing tastes and odors in drinking water. Water Sci. Technol. 40 (6), 279-285.

Young, W.F., Horth, H., Crane, R., Ogden, T., Arnott, M., 1996. Taste and odour threshold concentrations of potential potable water contaminants. Water Res. 30 (2), 331-340

Yu, J.W., Zhao, Y.M., Yang, M., Lin, T.F., Guo, Z.H., Gu, J.N., Li, S., Han, W., 2009. Occurrence of odour-causing compounds in different source waters of China. J. Water Supply Res Technol. 58 (8), 587-594.

Zhang K... Lin, T.F., Zhang T.Q. Li, C., Gao, N.Y., 2013. Characterization of typical taste and odor compounds formed by Microcystis aeruginosa. J. Environ. Sci. 25 (8), 1539-1548. 